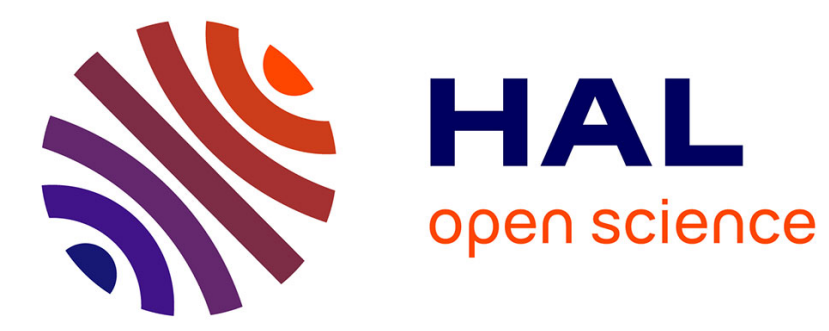

\title{
Consistency-Based Reliability Assessment
}

Laurence Cholvy, Laurent Perrussel, William Raynaut, Jean-Marc Thévenin

\section{To cite this version:}

Laurence Cholvy, Laurent Perrussel, William Raynaut, Jean-Marc Thévenin. Consistency-Based Reliability Assessment. European Conference on Symbolic and Quantitative Approaches to Reasoning with Uncertainty (ECSQARU 2015), Jul 2015, Compiègne, France. pp. 246-256. hal-01316842

\section{HAL Id: hal-01316842 \\ https://hal.science/hal-01316842}

Submitted on 17 May 2016

HAL is a multi-disciplinary open access archive for the deposit and dissemination of scientific research documents, whether they are published or not. The documents may come from teaching and research institutions in France or abroad, or from public or private research centers.
L'archive ouverte pluridisciplinaire HAL, est destinée au dépôt et à la diffusion de documents scientifiques de niveau recherche, publiés ou non, émanant des établissements d'enseignement et de recherche français ou étrangers, des laboratoires publics ou privés. 


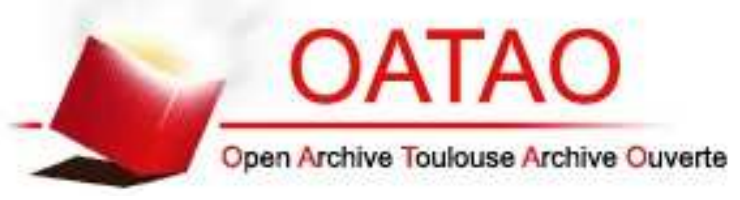

\section{Open Archive TOULOUSE Archive Ouverte (OATAO)}

OATAO is an open access repository that collects the work of Toulouse researchers and makes it freely available over the web where possible.

This is an author-deposited version published in : http://oatao.univ-toulouse.fr/ Eprints ID : 15443

The contribution was presented at:

https://ecsqaru2015.hds.utc.fr/

To cite this version : Cholvy, Laurence and Perrussel, Laurent and Raynaut, William and Thévenin, Jean-Marc Consistency-Based Reliability Assessment. (2015) In:

European Conference on Symbolic and Quantitative Approaches to Reasoning with Uncertainty (ECSQARU 2015), 15 July 2015 - 17 July 2015 (Compiègne, France).

Any correspondence concerning this service should be sent to the repository administrator: staff-oatao@listes-diff.inp-toulouse.fr 


\title{
Consistency-Based Reliability Assessment
}

\author{
Laurence Cholvy ${ }^{1}$, Laurent Perrussel ${ }^{2}$, \\ William Raynaut ${ }^{1,2}$, and Jean-Marc Thévenin ${ }^{2}$ \\ 1 ONERA Toulouse, France \\ laurence.cholvy@onera.fr \\ 2 IRIT-Université Toulouse 1 Capitole, Toulouse, France \\ laurent.perrussel@irit.fr, william.raynaut@irit.fr, \\ jean-marc.thevenin@irit.fr
}

\begin{abstract}
This paper addresses the question of assessing the relative reliability of unknown information sources. We propose to consider a phase during which the consistency of information they report is analysed, whether it is the consistency of each single report, or the consistency of a report w.r.t. some trusted knowledge or the consistency of different reports together. We adopt an axiomatic approach by first giving postulates which characterize how the resulting reliability preorder should be; then we define a family of operators for building this preorder and demonstrate that it satisfies the proposed postulates.
\end{abstract}

\section{Motivation}

Techniques for merging raw information have been studied in an extensive way. These techniques usually assume that all information provided by the sources (i.e. agents) should be considered as a whole. Two different approaches have been studied: the first one considers sources in an equal way and has led to merging techniques such as majority, arbitration merging or distance-based merging for solving conflict between contradicting information $[9,5,6]$. The second one distinguishes sources through a reliability criterion. Taking sources reliability into account provides rationales for discounting or ignoring pieces of information whose source is not considered as sufficiently reliable. Some promote a quantitative model of reliability: information sources are associated with a reliability level represented by a number used by the merging operator. According to the belief function theory, the reliability level of a source is a number between 0 and 1 . This number is then used by the discounting rule in order to weaken the importance of information provided by this source [13]. Some others promote a qualitative approach to reliability and consider that information sources are ranked according to their reliability. This order or pre-order is then used by the merging operator. In [3], the author defines a merging operator which assumes that the sources are totally ordered : if $s$ is said to be more reliable than $s^{\prime}$ and together provide contradicting information, then information provided by $s$ is privileged; while information provided by $s^{\prime}$ which does not contradict information of $s$ is also considered as acceptable. The same idea is followed by [10] for reasoning about more complex beliefs and in [12] for revising a belief base. 
All previous works assume that the reliability of the sources is given as a parameter (quantitative or qualitative): they do not address the question of how to build up this reliability.

This paper addresses this key question. We adopt a qualitative point of view to reliability representation : the relative reliability of information sources is represented by a total preorder. We focus on the question of estimating this reliability preorder in the following context: sources are unknown (no extra information about them is available) and information provided by the sources is only qualitative (i.e., statements). We propose to consider a phase, before the information merging phase, during which information sources are observed in order to obtain a reliability preorder. We consider that during this phase, the most important is to analyse the consistency of information reported by the different sources, be it the consistency of each single report, or the consistency of a report w.r.t. some trusted knowledge, or the consistency of different reports together.

This paper is organized as follows. Section 2 presents preliminary definitions. Postulates which axiomatically characterize the reliability preorders are given in section 3. Section 4 describes a generic operator building such preorders and demonstrate that it satisfies the postulates. Examples of operators are given in section 5. Finally, section 6 concludes the paper.

\section{Preliminaries}

Let $A$ be a finite set of agents; let $L$ be a propositional logic defined over a finite set of propositional letters and propositional constants $\top$ and $\perp$. An interpretation $m$ is a mapping from the set of formulas of $L$ to the set of truth values $\{0,1\}$ so that $m(\top)=1$ and $m(\perp)=0$. The set of all interpretations is denoted $M$. Interpretation $m$ is a model of formula $F$ iff $m(F)=1$. Tautologies are formulas which are interpreted by 1 in any interpretation. We write $\models F$ when $F$ is a tautology. A formula is consistent iff it has at least one model.

Let $\leq$ be a total preorder on $A$ representing the relative reliability of agents: $a \leq b$ stands for $b$ is at least as reliable as $a . G T(a, \leq)=\{x \in A \backslash\{a\}: a \leq x\}$ is the set of agents which are as least as reliable as $a$. Let $a \in A, \leq_{1}$ be a total preorder on $A$ and $\leq_{2}$ a total preorder on $A \backslash\{a\} ; \leq_{1}$ is compatible with $\leq_{2}$ iff $\forall x \forall y \quad x \leq_{2} y \Longrightarrow x \leq_{1} y$.

In the following, raw information is a communication consisting of a pair associating an agent and a statement:

- A communication set on $A$ is a set of pairs $\langle a, \varphi\rangle$ where $a \in A$ and $\varphi$ is a formula of $L .\langle a, \varphi\rangle$ means that agent $a$ has reported $\varphi$.

- Let $\Psi$ be a communication set on $A$. $A g(\Psi)=\{a \in A, \exists \varphi<a, \varphi>\in \Psi\}$.

- Given a communication set $\Psi$, we define the communication set of a as $\Psi_{a}=$ $\{\langle a, \varphi\rangle \mid\langle a, \varphi\rangle \in \Psi\}$ and the communication of a set of agents $C$ as

$$
\Psi(C)=\bigcup_{a \in C} \Psi_{a}
$$


The report associated to some $\Psi$ represents the content of the communication:

$$
\operatorname{Report}(\Psi)= \begin{cases}\bigwedge_{<a, \varphi>\in \Psi} \varphi & \text { if } \Psi \neq \emptyset \\ \top & \text { otherwise }\end{cases}
$$

- Let $\Psi$ and $\Psi^{\prime}$ be two communication sets on $A . \Psi$ and $\Psi^{\prime}$ are equivalent iff for any agent $a$ in $A$ : $\models \operatorname{Report}\left(\Psi_{a}\right) \leftrightarrow \operatorname{Report}\left(\Psi_{a}^{\prime}\right)$. That is, $a$ 's report in $\Psi$ is equivalent to $a$ 's report in $\Psi^{\prime}$. We write $\Psi \equiv \Psi^{\prime}$. Obviously $\Psi \equiv \Psi^{\prime}$ iff $\forall C \subseteq A \quad \Psi(C) \equiv \Psi^{\prime}(C)$

- Let $\Psi$ and $\Psi^{\prime}$ be two communication sets on $A . \Psi$ and $\Psi^{\prime}$ are weakly equivalent iff for any agent $a$ in $A, \exists b, \exists c \in A$ such that $\models \operatorname{Report}\left(\Psi_{a}\right) \leftrightarrow \operatorname{Report}\left(\Psi_{b}^{\prime}\right)$ and $\models \operatorname{Report}\left(\Psi_{a}^{\prime}\right) \leftrightarrow \operatorname{Report}\left(\Psi_{c}\right)$. That is, we relax here the constraint that report of agent $a$ should be equivalent both in $\Psi$ and $\Psi^{\prime}$; instead we only require some other agent, possibly different from $a$, report equivalent information. We write $\Psi \rightleftharpoons \Psi^{\prime}$. Obviously $\Psi \equiv \Psi^{\prime}$ iff $\forall C \subseteq A \quad \Psi(C) \rightleftharpoons \Psi^{\prime}(C)$

\subsection{IC-contradictory communication sets}

Consistency of reports will be evaluated with respect to some integrity constraint $I C$, a consistent formula of $L$. $I C$ has to be viewed as information taken for granted or certain. Let us now revisit the classical notion of minimal inconsistent set w.r.t. communication sets. Formally, let $\Psi$ be a set of communications on $A$ :

- $\Psi$ is $I C$-contradictory iff $\operatorname{Report}(\Psi) \wedge I C$ is inconsistent; otherwise $\Psi$ is $I C$ consistent.

- $\Psi$ is minimal $I C$-contradictory iff $\Psi$ is $I C$-contradictory and no strict subset of $\Psi$ is $I C$-contradictory.

- $\Psi \perp I C$ denotes the set of minimal IC-contradictory subsets of $\Psi$.

- $A^{\perp}=\cup_{F \in \Psi \perp I C} A g(F)$ is the set of agents which have reported a piece of information which belongs to some minimal $I C$-contradictory communication set. Notice that $A^{\perp} \neq \emptyset$ iff $\Psi$ is $I C$-contradictory.

Example 1. Consider agents $a, b, c, d$ and propositional letters $p, q, r, s$. Assume $I C=\neg(p \wedge q)$ and $\Psi=\{\langle a, p\rangle,\langle a, r\rangle,\langle b, q\rangle,\langle c, \neg r\rangle,\langle d, s\rangle\}$. The minimal $I C$-contradictory subsets of $\Psi$ are $\left.E_{1}=\{\langle a, p\rangle,<b, q\rangle\right\}$ and $E_{2}=\{\langle c, \neg r\rangle,\langle a, r\rangle\}$. Thus $A^{\perp}=\{a, b, c\}$.

Example 2. Consider now agents $a, b$ and propositional letters $p, q$. Assume $I C=$ $p$ and $\Psi=\{<a, \neg p \wedge q\rangle,<b, \neg q\rangle\}$. The $I C$-contradictory subsets of $\Psi$ are $E_{1}=\{\langle a, \neg p \wedge q\rangle\}$ and $\left.E_{2}=\{\langle a, \neg p \wedge q\rangle,<b, \neg q\rangle\right\}$ but only $E_{1}$ is minimal. Thus $A^{\perp}=\{a\}$.

This last example shows that $A^{\perp}$ is not the set of all agents which bring some contradiction. $A^{\perp}$ is to be seen as the set of agents which prevent the consistency of $\Psi$ i.e if communications of agents of $A^{\perp}$ are ignored, $\Psi$ becomes $I C$-consistent. I.e., $\Psi \backslash A^{\perp}$ is $I C$-consistent (but not necessarly maximal consistent). 


\section{$2.2 \quad I C$-conflicting agents}

We go further for revisiting the notion of minimal inconsistent set by considering set of agents rather than set of statements. This set helps us to identify the sources which are related to inconsistent report.

- Let $C \subseteq A$. $C$ is $I C$-conflicting iff $\operatorname{Report}(\Psi(C)) \wedge I C$ is inconsistent.

- $C$ is minimal IC-conflicting iff it is $I C$-conflicting and no strict subset of $C$ is $I C$-conflicting.

Example 3. Let's consider the previous example. $\{a, b, c, d\}$ is $I C$-conflicting. $\{a, b\}$ and $\{a, c\}$ are minimal $I C$-conflicting.

We can show that the union of minimal $I C$-conflicting subsets of $A$ is included in $A^{\perp}$. But the reverse is not true: consider $I C=\neg q$ and $\Psi=\{\langle a, p\rangle,<$ $b, \neg p>,<b, q>\} . A^{\perp}=\{a, b\}$ while the only minimal $I C$-conflicting subset of agents is $\{b\}$.

\section{Assessing reliability}

The following postulates define in an axiomatic way that reliability assessment should be rooted in the notion of contradiction occurring in a set of communications.

Given a set of agents $A$, an integrity constraint $I C$ and a communication set $\Psi$, the total preorder representing the relative reliability of agents in $A$ is denoted $\Gamma^{I C, A}(\Psi)$. The operator $\Gamma$, which defines this relative reliability preorder is characterized as follows:

P1 $\Gamma^{I C, A}(\Psi)$ is a total preorder on $A$.

P2 If $\Psi \equiv \Psi^{\prime}$ then $\Gamma^{I C, A}(\Psi)=\Gamma^{I C, A}\left(\Psi^{\prime}\right)$.

P3 If $\models I C \leftrightarrow I C^{\prime}$ then $\Gamma^{I C, A}(\Psi)=\Gamma^{I C^{\prime}, A}(\Psi)$.

P4 If $\models \operatorname{Report}\left(\Psi_{a}\right)$ then $\Gamma^{I C, A}(\Psi)$ is compatible with $\Gamma^{I C, A \backslash\{a\}}\left(\Psi \backslash \Psi_{a}\right)$.

P5 If $A$ is not $I C$-conflicting then $\Gamma^{I C, A}(\Psi)$ is the equality preorder.

P6 If $A$ is $I C$-conflicting then $A \backslash A^{\perp} \subseteq G T\left(a, \Gamma^{I C, A}(\Psi)\right)$ for any $a \in A^{\perp}$.

P7 If $\left\{a_{1}, \ldots, a_{k}\right\}(k \geq 2)$ is a minimal $I C$-conflicting subset of agents, then $\exists i \forall j j \neq i, G T\left(a_{j}, \Gamma^{I C, A}(\Psi)\right) \subset G T\left(a_{i}, \Gamma^{I C, A}(\Psi)\right)$.

Postulate $\mathbf{P} 1$ specifies that the expected result is a total preorder. P2 and P3 deal with syntax independence. More precisely, if we consider two equivalent communication sets or if we consider two equivalent $I C$ formulas, then we get the same total preorder on agents. $\mathbf{P} 4$ states that an agent which reports a tautology or which reports no information has no influence on the relative reliability of other agents. P5, P6 and P7 focus on consistency of information provided by agents in $A$. Postulate P5 considers the case when $A$ is not $I C$-conflicting (i.e $\Psi$ set is not $I C$-contradictory). In such a case, the sources are considered as equally reliable. $\mathbf{P} \mathbf{6}$ and $\mathbf{P 7}$ consider the cases when $A$ is $I C$-conflicting. According to P6, any agent reporting a piece of information belonging to some 
minimal $I C$-contradictory communication set is considered as less reliable than any other agent which have not. According to P7, if some agents are minimally $I C$-conflicting, then at least one of these agents is strictly less reliable than the others. This is inline with our understanding of reliability: if some agents are equally reliable, then after merging we will believe, with the same strength, information they will provide. However, it is generally assumed $[4,11]$ that graded belief satisfies a modal logic axiom which states that beliefs should be consistent: that is, two contradictory pieces of information cannot be believed with the same strength. Consequently, agents who are involved in a minimal $I C$-conflicting set cannot be equally reliable.

\section{Operator assessing reliability}

In this section, we propose a generic operator which builds the reliability preorder of agents by taking into account their contribution to inconsistencies.

\subsection{Contribution of agents to inconsistencies}

We start by introducing a measure to quantify the inconsistency degree of communication sets w.r.t. some $I C$. This measure is adapted from the one proposed in [8] for measuring inconsistency of sets of formulas.

Definition 1. A syntax weak-independent $I C$-inconsistency measure is a function $I_{I C}$ which associates any communication set $\Psi$ with a positive real number $I_{I C}(\Psi)$ so that:

- Consistency : $I_{I C}(\Psi)=0$ iff $\Psi$ is IC-consistent.

- Monotony : $I_{I C}\left(\Psi \cup \Psi^{\prime}\right) \geqslant I_{I C}(\Psi)$

- Dominance : for all $\phi$ and $\psi$, if $I C \wedge \phi \models \psi$ and $I C \wedge \phi$ is consistent, then $\left.\left.I_{I C}(\Psi \cup\{<a, \phi\rangle\}\right) \geqslant I_{I C}(\Psi \cup\{<b, \psi\rangle\}\right)$ for any $a, b \in A$.

- Free formula independence : If $\langle a, \phi\rangle$ is free (it does not belong to any minimal $I C$-contradictory subset of $\Psi \perp I C)$, then $I_{I C}(\Psi)=I_{I C}(\Psi \backslash\{<$ $a, \phi>\})$.

- Syntax weak-independence :

1. for all $I C^{\prime}$ if $\models I C \leftrightarrow I C^{\prime}$ then $I_{I C}(\Psi)=I_{I C^{\prime}}(\Psi)$

2. for all $\Psi^{\prime}$ if $\Psi \rightleftharpoons \Psi^{\prime}$ then $I_{I C}(\Psi)=I_{I C}\left(\Psi^{\prime}\right)$

The consistency property states that the measure of inconsistency of a communication set is null iff this communication set is not $I C$-contradictory. The monotony property says that the measure of inconsistency of a communication set does not decrease if we add more communications in this set. The dominance property states that logically stronger reports bring potentially more contradictions. The free formula independence property states that adding a report that does not cause any contradiction cannot change the consistency measure of the communication set. Finally, the syntax weak-independence says that the measure of inconsistency of a communication set does not depend on the syntax on 
the integrity constraints. It also says that two weakly equivalent communication sets get the same measure of inconsistency.

Notice that $I_{I C}(\emptyset)=0$ since $\operatorname{Report}(\emptyset)=\top$.

Proposition 1. Let $\Psi$ be a communications set on $A, I C$ an integrity constraint and $a \in A$. If $a \notin A^{\perp}$ then $I_{I C}\left(\Psi_{a}\right)=0$. The reverse is not true.

Next we consider a function for measuring how much an agent contributes to the $I C$-inconsistency of a communication set. The contribution of an agent to the fact that $\Psi$ is $I C$-contradictory is defined as the Shapley value and measures the importance of this agent in a coalitional game defined by function $I_{I C}$ [8].

Definition 2. Consider a set of agents $A$, a communication set $\Psi$ on $A$, an integrity constraint $I C$ and a syntax weak-independent $I C$-inconsistency measure $I_{I C}$. Function $\operatorname{Cont}_{\Psi}^{I_{I C}}$ associates any agent a with a positive real number $\operatorname{Cont}_{\Psi}^{I_{I C}}(a)$ so that:

$$
\operatorname{Cont}_{\Psi}^{I_{I C}}(a)=\sum_{\substack{C \subseteq A \\ C \neq \emptyset}} \frac{(|C|-1) !(|A|-|C|) !}{|A| !}\left(I_{I C}(\Psi(C))-I_{I C}(\Psi(C \backslash\{a\}))\right)
$$

Proposition 2. Let $\Psi$ be a communications set on $A, I C$ an integrity constraint and $a \in A$. Then, $a \notin A^{\perp} \Longrightarrow \operatorname{Cont}_{\Psi}^{I_{I C}}(a)=0$. The reverse is not true.

Given the function $\operatorname{Cont}_{\Psi}^{I_{I C}}$, one can obviously define a total preorder on $A$ as follows:

Definition 3. Let $a$ and $b$ be two agents of $A$.

$$
a \leq_{C o n t}^{I_{\Psi C}} b \quad \text { iff } \quad \operatorname{Cont}_{\Psi}^{I_{I C}}(a) \geq \operatorname{Cont}_{\Psi}^{I_{I C}}(b)
$$

This defines the reliability as follows: a source is considered strictly more (resp, equally) reliable than another iff its contribution to the global inconsistency is stricty smaller than (resp equal to) the contribution of the other. But, unfortunately, this preorder does not satisfy the seven postulates, as shown in the following.

Proposition 3. $\leq_{\text {Cont } I_{\Psi}^{I_{I C}}}$ satisfies $\mathbf{P} \mathbf{1}-\mathbf{P} \mathbf{6}$ but does not satisfy $\mathbf{P} 7$.

To prove that $\leq_{C o n t_{\Psi}^{I C}}$ does not satisfy $\mathbf{P} 7$, just consider $\Psi=\{<a, p\rangle$, $<b, \neg p>\}$ and $I C=$ true. $\{a, b\}$ is a minimal IC-conflicting set of agents but however, $a={ }_{C o n t}^{I_{I C}} b$.

As a consequence, we have to find another way to buid operators for reliability assessment. This is the purpose of the following paragraph. 


\section{$4.2 \quad \Gamma^{I_{I C}}$ operator}

Definition 4. Consider a set of agents A, a communication set on A, $\Psi$, an integrity constraint $I C$ and a given syntax weak-independent $I C$-inconsistency measure $I_{I C}$. The operator $\Gamma^{I_{I C}}$ for assessing reliability is defined by:

1. $X \leftarrow A$

2. $E \leftarrow \Psi \perp I C$

3. $\leq \leftarrow\{a \leq b \mid a, b \in A\}$

4. while $E \neq \emptyset$ do

(a) Deterministically choose $a \in A g\left(\cup_{F \in E} F\right)$ which maximizes $\operatorname{Cont}_{\Psi}^{I_{I C}}(a)$

(b) $X \leftarrow X \backslash\{a\}$

(c) $E \leftarrow E \backslash\{F \in E \mid a \in A g(F)\}$

(d) $\leq \leftarrow \leq \backslash\{b \leq a \mid b \in X\}$

\section{5. return $\leq$}

In the previous algorithm, $X$ is the set of agents which has to be ordered, $E$ is the set of minimal $I C$-contradictory subsets of $\Psi$ which contain communications of agents in $X . \leq$ is the reliability pre-order and $a \leq b$ stands for $b$ is at least as reliable as $a$. First Lines $1-3$ initialize the variables: $X$ is initialized as $A, E$ contains all the minimal $I C$-contradictory subsets of $\Psi$ and $\leq$ is equality. Then according to lines (4) and (a)-(d), the operator chooses one agent $a$ among those which maximally contribute to the $I C$-contradiction of $\Psi$, removes $a$ from $X$, deletes from $E$ all the subsets which contains some communication of $a$, and updates $\leq$ so that $a$ is no more reliable than agents in $X$. This is done until $E$ is empty. Notice that line (a) expresses a deterministic choice, such as lexicographic order.

Example 4. Consider agents $a, b, c, d$ and propositional letters $p, q, r$. Consider $\Psi=\{\langle a, p\rangle,\langle b, q\rangle,\langle c, \neg q\rangle,\langle d, r\rangle\}$ and $I C=\neg r$. Consequently, $\Psi \perp I C=\{\{<b, q>,<c, \neg q\rangle\},\{<d, r>\}\}$. Assume that:

$$
\operatorname{Cont}_{\Psi}^{I_{I C}}(a)<\operatorname{Cont}_{\Psi}^{I_{I C}}(b)=\operatorname{Cont}_{\Psi}^{I_{I C}}(c)<\operatorname{Cont}_{\Psi}^{I_{I C}}(d)
$$

Assume a lexicographic order for choice. First, Lines $1-3$ sets $X, E$ and $\leq$ as follows:

$$
\begin{aligned}
& X=\{a, b, c, d\} \quad E=\{\{<b, q>,<c, \neg q>\},\{<d, r>\}\} \\
& \leq=\{a=b=c=d\}
\end{aligned}
$$

Next, first iteration chooses " $d$ " at step $(a)$ and we get:

$$
\begin{aligned}
& X=\{a, b, c\} \quad E=\{\{<b, q>,<c, \neg q>\}\} \\
& \leq=\{d<a=b=c\}
\end{aligned}
$$

Lexicographic order entails that the 2 nd iteration chooses " $b$ ":

$$
\begin{aligned}
& X=\{a, c\} \quad E=\emptyset \\
& \leq=\{d<b<a=c\}
\end{aligned}
$$

As $E=\emptyset$, the algorithm stops and returns the pre-order: $d<b<a=c$. 
The following propositions show that ranks are coherent with the inconsistency measure. That is, the more an agent is inconsistent, the less it is reliable:

Proposition 4. Let $\Psi$ be a communications set on $A$ and $I C$ an integrity constraint. Let $\leq$ be the preorder given by operator $\Gamma_{1}^{I_{I C}}$. For any two agents a and $b \in A$ :

$$
\text { If } \operatorname{Cont}_{\Psi}^{I_{I C}}(a) \geqslant \operatorname{Cont}_{\Psi}^{I_{I C}}(b) \text { then } a \leq b
$$

We have the immediate following corollary:

Corollary 1. If $a \notin A^{\perp}$ then for all $b \in A, b \leq a$.

The opposite direction of previous proposition can only be considered for strict order. This is due to the choice step (a): an agent may maximize the consistency measure but may not be chosen; agents may then be considered with same rank of reliability as agents getting a lower measure related to their contribution to inconsistency. In the previous example, $a=c$ while $\operatorname{Cont}_{\Psi}^{I_{I C}}(a)<\operatorname{Cont}_{\Psi}^{I_{I C}}(c)$.

Proposition 5. Let $\Psi$ be a communications set on $A$ and $I C$ an integrity constraint. Let $\leq$ be the preorder given by operator $\Gamma_{1}^{I_{I C}}$. For any two agents a and $b \in A$ :

$$
\text { If } a<b \text { then } \operatorname{Cont}_{\Psi}^{I_{I C}}(a) \geq \operatorname{Cont}_{\Psi}^{I_{I C}}(b)
$$

The two previous propositions show that the choice step plays a crucial role in the behavior in the operator. Indeed this choice enforces the satisfaction of postulate $\mathbf{P 7}$.

Theorem 1. Consider a set of agents $A$, a communication set on $A, \Psi$, an integrity constraint IC and a syntax weak-independent $I C$-inconsistency measure $I_{I C} . \Gamma^{I_{I C}}$ operators satisfy postulates $\mathbf{P 1 - P 7}$.

\section{$5 \quad$ Examples of Inconsistency measures}

Let us now detail two possible inconsistency measures which allow us to build two reliability assessment operators. These two measures are based on the ones proposed by [8]. The first measure is inspired by the drastic distance:

Definition 5 (Drastic measure). Let $\Psi$ be a set of communications on $A$ and $I C$ a consistent formula. The drastic inconsistency measure $I_{d}^{I C}$ is defined by:

$$
I_{d}^{I C}(\Psi)= \begin{cases}0 & \text { if } \Psi \text { is } I C \text {-consistent } \\ 1 & \text { otherwise }\end{cases}
$$

Theorem 2. $I_{d}^{I C}$ is a syntax weak-independent inconsistency measure.

Then $\Gamma_{d}^{I C}$ is a good candidate to assess reliability. 
Example 5. Consider agents $a, b, c, d$ and propositional letters $p, q, r$. Consider $\Psi=\{\langle a, p \wedge q\rangle,\langle b, q\rangle,\langle c, \neg q\rangle,\langle d, r\rangle\}$ and $I C=\neg r$. Then we get: $\operatorname{Cont}_{\Psi}^{I_{d}^{I C}}(a)=\frac{1}{12} \quad \operatorname{Cont}_{\Psi}^{I_{d}^{I C}}(b)=\frac{1}{12} \quad \operatorname{Cont}_{\Psi}^{I_{d}^{I C}}(c)=\frac{3}{12} \quad \operatorname{Cont}_{\Psi}^{I_{d}^{I C}}(d)=\frac{7}{12}$. Operator $\Gamma^{I^{I C}}$ returns $d<c<a=b$

Let us now consider a second measure which is more refined than Drastic measure. The measure is based on minimal inconsistency communication sets and the intuition is that the degree of inconsistency is proportional to the number of inconsistent subsets. The measure has to take care of our syntax-based perspective: to avoid syntactic biases, the measure considers the whole set of communications given by an agent.

Definition 6 (Minimal Inconsistent subsets measure). Let $\Psi$ be a set of communications on $A$ and $I C$ a consistent formula. The inconsistency measure $I_{M I}^{I C}$ based on the number of minimal IC-contradictory subset is defined as:

$$
I_{M I}^{I C}(\Psi)=\left|\left(\bigcup_{a \in A g(\Psi)}<a, \operatorname{Report}\left(\Psi_{a}\right)>\right) \perp I C\right|
$$

Theorem 3. $I_{M I}^{I C}$ is a syntax weak-independent inconsistency measure.

Then $\Gamma_{M I}^{I C}$ is a good candidate to assess reliability. Let's illustrate this second measure on the same example.

Example 6. Consider agents $a, b, c, d$ and propositional letters $p, q, r$. Consider $\Psi=\{\langle a, p \wedge q\rangle,\langle b, q\rangle,\langle c, \neg q\rangle,\langle d, r\rangle\}$ and $I C=\neg r$. Then we get: $\operatorname{Cont}_{\Psi}^{I_{M I}^{I C}}(a)=\frac{1}{2} \quad \operatorname{Cont}_{\Psi}^{I_{M I}^{I C}}(b)=\frac{1}{2} \quad \operatorname{Cont}_{\Psi}^{I_{M I}^{I C}}(c)=1 \quad \operatorname{Cont}_{\Psi}^{I_{M I}^{I C}}(d)=1$. Notice that the contributions of $c$ and $d$ are equal. According to these contributions $\Gamma^{I_{M I}^{I C}}$ return either $d<c<a=b$ or $c<d<a=b$ depending on the deterministic choice.

\section{Conclusion}

This work proposes to assess the relative reliability of some information sources by analysing the consistency of information they report, whether it be the consistency of each single report, or the consistency of a report as regard to some trusted knowledge or the consistency of different reports together. We have given some postulates stating what the relative reliability preorder should be. Then we have introduced a generic operator for building such preorder which is parametrized by a function for measuring the inconsistency of the information reported. We prove that this generic operator agrees with the postulates.

This framework may be extended in several ways. First, inconsistency measures should deserve more attention. Recent work on this topic [7,1] shows promising results such as giving a weight to the inconsistency itself. A second 
issue concerns our key principle relying assessment considering only inconsistency: the more agent is connected to inconsistency, the less it is reliable. The reverse notion, might then also be considered for assessing the reliability. In other words, how can we "reward" an agent which is never inconsistent. The third issue concerns the one shot dimension of the assessment process: iteration should be possible and reliability assessment should then be viewed as a refinement process. In that case, the key issue is to set rationales for changing reliability from $a<b$ to $b<a$. Finally, it must be noticed that if one has already some partial information about the reliability of the agents (for instance, one knows that agent $a$ is more reliable than $b$ but has no idea about $c$ reliability) then the process described in this paper is not applicable as is: in this case, we could achieve reliability assessment by combining that preorder with the one produced by the operator $\Gamma^{I_{I C}}$. For future work, we plan to study these agregation operators.

As we can see the proposed framework offers numerous perspectives and our aim is to take advantage of its flexibility for going further.

Acknowledgements. We sincerely thank the anonymous reviewers whose questions helped us to improve the paper.

\section{References}

1. Besnard, P. Revisiting Postulates for Inconsistency Measures. Proc. of JELIA 2014, Funchal, Portugal, September 2014.

2. V. Borja Macías, P. Pozos Parra: Model-based belief merging without distance measures. Proc. of AAMAS 2007, Honolulu, USA, May 2007.

3. L. Cholvy, Reasoning about merged information, In Handbook of Defeasible Reasoning and Uncertainty management, Vol 1, Kluwer Publishers 1998.

4. R. Demolombe, C-J. Liau. A logic of Graded Trust and Belief Fusion. Proc. of the $4^{\text {th }}$ Workshop on Deception, Fraud and Trust in Agent Societies, Montréal, Québec, 2001.

5. S. Benferhat, D. Dubois, S. Kaci, H. Prade: Possibilistic Merging and DistanceBased Fusion of Propositional Information. Annals of Mathematics and Artificial Intellgence 34(1-3): 217-252

6. P. Everaere, S. Konieczny, P. Marquis: Disjunctive merging: Quota and Gmin merging operators. Artificial Intelligence 174(12-13): 824-849 (2010)

7. John Grant, Anthony Hunter: Distance-Based Measures of Inconsistency. Proc.of ECSQARU 2013, Utrecht, The Netherlands, 2013.

8. Anthony Hunter, Sébastien Konieczny: On the measure of conflicts: Shapley Inconsistency Values. Artif. Intell. 174(14): 1007-1026 (2010)

9. S. Konieczny, R. Pino Pérez: Merging Information Under Constraints: A Logical Framework. Journal of Logic and Computation 12(5): 773-808 (2002)

10. C.-J. Liau, A modal logic framework for multi-agent belief fusion, In ACM Transactions on Computational Logic, 6(1): 124-174 (2005)

11. N. Laverny and J. Lang. From Knowldege-based programs to graded belief-based programs, Part I: on-line reasoning. Synthese 147, pp 277-321, Springer, 2005.

12. E. Lorini, L. Perrussel, J.M. Thévenin, A Modal Framework for Relating Belief and Signed Information. Proc. of CLIMA'11, Springer-Verlag, 2011.

13. G. Shafer, A mathematical Theory of Evidence, Princeton University Press, 1976. 